\title{
Editorial
}

\section{Gut Microbiota and Personalized Nutrition}

Jing $X$. Kang

Massachusetts General Hospital and Harvard Medical School, Boston, Mass., USA

The gut microbiota has recently attracted unprecedented attention from the biomedical community for the mounting evidence supporting its central role in human health and disease [1]. The gut microbiota consists of the trillions of bacterial microorganisms, spanning over 500 species, that inhabit our gastrointestinal tracts. We now know that these bacteria are not simply commensal organisms in our bodies, but instead serve as an important 'organ' that regulates metabolic processes, including the digestion and absorption of nutrients, synthesis of vitamins, modulation of mucosal immunity, as well as production of toxins and carcinogens $[1,2]$. The composition of the gut microbial community - whether the bacterial numbers are balanced or in dysbiosis - determines the nature of its influence on numerous physiological and pathological conditions. Since the gut microbial profile is unique to each individual, evolves over a lifetime, and can be altered by internal and external factors (especially the diet), the gut microbiome presents us with further complexities as well as new opportunities for nutrigenomics and personalized nutrition.

When we study the effects of dietary nutrients on gene expression, we must be aware that these effects can be mediated by gut bacteria. The gut microbiota can influence gene expression both locally and systemically. Through interactions with the intestinal mucosal tissue, certain species of bacteria can influence the expression of genes involved in nutrient absorption or immune function [3], while some substances generated by gut bacteria can enter the bloodstream; for example, short-chain fatty acids (produced by fermentation of polysaccharides) can regulate lipogenesis gene expression in the liver, and toxins like lipopolysaccharides (LPS) can affect inflammation-related genes [4]. The quantities of these bacteria, such as the LPS-producing Escherichia coli, can be significantly altered by dietary nutrients. For example, a diet high in saturated fat has been shown to increase E. coli and subsequent LPS production [5], while a laminarin-supplemented diet can suppress E. coli numbers and result in lower levels of inflammatory cytokines [6]. These findings illustrate a new interface for the regulation of gene expression by dietary nutrients, and also reveal an alternative pathway for regulating gene expression through the manipulation of the gut microbiota. 
The recent advances in gut microbiota research emphasize the potential of modifying gut bacteria composition as a novel, effective intervention for disease management and health promotion. Alteration of gut microbiota composition has been implicated in a number of diseases. For instance, an increased ratio of the phylum Firmicutes to the genus Bacteroides has been linked to obesity [4]. However, our understanding of the relationship between gut microbiota and disease is still limited. Further investigation is needed to elucidate how alterations within the microbiome can contribute to metabolic diseases and address key questions, such as: what role specific bacteria play within the gut, what the ideal ratios between certain types of bacteria are, how they interact with each other and with host tissues, how they affect nutrient metabolism, and so on. This knowledge will enable us to characterize gut microbiomes for different health conditions. Efforts must also be devoted to identifying gut microbiota biomarkers, such as specific bacterial gene expression or the lack of a certain bacterial species that can determine someone's risk for developing a metabolic disease.

It is essential that we recognize the interplay among the diet, gut microbiota, and the host. Developing dietary interventions based on one's profile to optimize gut microbial composition can be an important practice for personalized nutrition. It is a top priority to examine the effects of dietary factors on the gut microbiota and identify those that can effectively modify the gut bacteria composition. Specifically, we need to understand which nutrients can increase the beneficial bacteria and which can suppress the harmful bacteria, so that we can formulate dietary regimens and food products that can be used to normalize gut microbial composition.

To integrate the gut microbiota into personalized nutrition, it is also important to develop technologies that allow us to quickly and accurately determine one's gut microbial profile. With this testing capability we will be able to readily monitor personal changes in gut bacteria composition during disease development and over the course of intervention. The information obtained through these measurements will help us evaluate the relationship between the gut microbiota and disease as well as the efficacy of intervention.

Given that the gut microbiota is emerging as a critical factor influencing human nutrition and metabolism, we must take into account the specifics of an individual's gut microbiome together with other personal information, including their diet, genome, proteome, and metabolome, in order to develop a more effective and beneficial approach to personalized nutrition.

\section{References}

- 1 Sekirov I, Russell SL, Antunes LC, Finlay BB: Gut microbiota in health and disease. Physiol Rev 2010;90: 859-904.

2 Guarner F, Malagelada JR: Gut flora in health and disease. Lancet 2003;361:512-519.

- 3 Kussmann M, Van Bladeren PJ: The extended nutrigenomics - understanding the interplay between the genomes of food, gut microbes, and human host. Front Genet 2011;2:1-13.

- 4 DiBaise JK, Zhang H, Crowell MD, Krajmalnik-Brown R, Decker GA, Rittmann BE: Gut microbiota and its possible relationship with obesity. Mayo Clin Proc 2008;83:460-469.

- 5 Kim KA, Gu W, Lee IA, Joh EH, Kim DH: High fat diet-induced gut microbiota exacerbates inflammation and obesity in mice via the TLR4 signaling pathway. PLoS One 2012;7:e47713.

- 6 Walsh AM, Sweeney T, O'Shea CJ, Doyle DN, O'Doherty JV: Effect of dietary laminarin and fucoidan on selected microbiota, intestinal morphology and immune status of the newly weaned pig. Br J Nutr 2013;26:1-9. 